\title{
NOTE ON CONTRACTION COEFFICIENTS OF JETS OF GAS
}

\author{
By Edgar Buckingham
}

ABSTRACT

1. A jet of liquid from a sharp-edged orifice contracts more than a jet of gas because the gas expands after leaving the orifice; and theoretical expressions for the ratio of the two contraction coefficients have hitherto been deduced from the assumption that the expansion is the same in all directions. In the present paper this is replaced by the assumption that the force exerted on the upstream face of an orifice plate on the end of a pipe depends only on the static pressure and the momentum of the approaching stream.

2. The resulting expression is compared with the experiments of Witte on water, of Bachmann on air, and recent unpublished experiments on natural gas. The conditions presupposed by the theory are strictly satisfied in Bachmann's experiments but in the others they are satisfied only when the ratio, $\beta$, of orifice to pipe diameter is small.

3. The conclusions are as follows:

(a) Bachmann's experiments on air at low differentials agree with Witte's on water within 0.5 per cent.

(b) The measurements on natural gas agree with Bachmann's on air within 0.5 per cent.

(c) As far as the critical pressure ratio, the new formula represents the results on natural gas within 0.5 per cent for $\beta=0.27$; within 0.3 per cent for $\beta=0.38$; and within 0.8 per cent for $\beta=0.5$. For $\beta=0.7$ the departure increases to nearly 4 per cent.

\section{CONTENTS}

I. Introduction

II. Development of a new formula

1. Conditions ....

2. Notation

3. Remarks_......... 767

4. Basis for a new treatment of the problem $\ldots \ldots$

5. Isentropic flow from $\left(p_{1}, \rho_{1}\right)$ to $p_{2} \ldots$

6. Flow at constant density from $\left(p_{1}, \rho_{1}\right)$ to $p_{3} \ldots \ldots 768$

7. Application of the fundamental assumption

III. Examination of the experimental data

IV. Comparison with the new formula

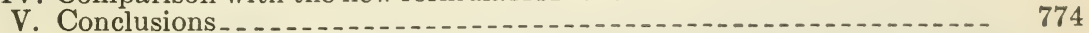

\section{INTRODUCTION}

The pressure in a jet of fluid issuing from a square-edged orifice in a thin plate does not fall to equality with the outside pressure until the filaments have become parallel. Between the plane of the orifice and the vena contracta there is a radial pressure gradient outward, and if the fluid is a gas it expands transversely as well as longitudinally, whereas in the case of a liquid there is no expansion. The cross section of the vena contracta is therefore larger with a gas than with a liquid.

The ratio of the two sectional areas would be given by a complete solution of the equations of motion, but until the mathematical 
difficulties of the problem have been overcome, any attempt to compute the ratio a priori must rest on simplifying, approximate assumptions. Two such computations have recently been made, ${ }^{12}$ both based on the assumption that the gas in the jet expands uniformly in all directions; but while the formulas obtained agree well with the experimental data with which they have been compared, the fundamental assumption has been criticized ${ }^{3}$ as very improbable under the conditions of acceleration and distortion prevailing in the jet. I have therefore thought it worth while to start from a different initial hypothesis and to compare the result obtained with certain additional experimental data which have not yet been published and so have not been available to previous writers.

\section{DEVELOPMENT OF A NEW FORMULA}

\section{CONDITIONS}

The orifice is to be a cylindrical hole in the center of a thin, flat diaphragm across the end of a straight pipe. The outside space into which the discharge takes place is to be so large that the static pressure in it is sensibly uniform everywhere outside the jet from the orifice; and the jet will be regarded as having a definite boundary, as far as the vena contracta, the frictional drag at the boundary being negligible. We consider only steady states of flow.

The fluid shall be some gas, such as air, which may be treated as subject to the ordinary ideal gas equations over the short range of pressure and density involved in flow through an orifice at jet speeds below the speed of sound in the jet.

The Reynolds number shall be high enough that the motion is no longer appreciably affected by viscosity, and that the velocity and static pressure are sensibly uniform all over the section at the vena contracta, the pressure there being the same in the jet as in the surrounding space.

\section{NOTATION}

The following notation will be adopted, all values being expressed in terms of a set of normal units, such as the cgs or the "British absolute" system.

$A=$ the area of cross section of the pipe;

$m A=$ the area of the orifice, $m$ being the area ratio and $\sqrt{m}=\beta$ the diameter ratio;

$p_{1}=$ the static pressure in the pipe ahead of the orifice;

$\rho_{1}=$ the density of the gas at that place;

$S_{1}=$ the linear speed of flow along the pipe;

$p_{2}=$ the pressure in the outside space;

$\rho_{2}=$ the density of the gas after isentropic expansion from $\left(p_{1}, \rho_{1}\right)$ to $p_{2}$;

$S_{2}=$ the speed at the vena contracta;

$\mu_{a}=$ the contraction coefficient, so that the area of the vena contracta is $\mu_{a} m A$;

$M=$ the mass flow; evidently

$$
M=\mu_{a} m A \rho_{2} S_{2}
$$

I R. Witte, Techn. Mech. u. Thermodyn., 1, p. 118; March, 1930.

d. Rupnel, Techin. Mech. u. Thermodyn., 1, p. 151; A pril, 1930.

A. Busomann, Techn. Mech. u. Thormodyn., 1, p. 338; September, 1930. 
$p_{3}=$ the outside pressure that would be required to produce this same mass flow, from the same initial pressure and density, if the density were kept constant at the value $\rho_{1}$, which could, in principle, be accomplished by withdrawing heat at a suitable rate instead of allowing the flow to be adiabatic;

$S_{3}=$ the speed at the vena contracta in this case; and

$\mu=$ the contraction coefficient. By the definition of $S_{3}$ and by (1) we have

$$
\mu m A \rho_{1} S_{3}=M=\mu_{a} m A \rho_{2} S_{2}
$$

$\gamma=C_{\mathrm{v}} / C_{\mathrm{v}}=$ the specific heat ratio of the gas;

$F_{\mathrm{o}}=$ the force exerted by the gas inside the pipe tending to push the orifice plate downstream off the end of the pipe. To simplify the appearance of the equations the following abbreviations will also be employed

$$
\left.\begin{array}{cc}
\frac{p_{2}}{p_{1}}=y & \frac{p_{3}}{p_{1}}=w \\
\frac{\gamma}{\gamma-1} y^{\frac{1}{\gamma}}\left(1-y^{\frac{\gamma-1}{\gamma}}\right)=\phi
\end{array}\right\}
$$

\section{REMARKS}

If the density does not change during a fall of pressure, compressibility does not come into play, so that its value is immaterial and the shape of the jet depends only on the value of the Reynolds number $R=\frac{D S_{\rho}}{\eta}$, in which $D$ is the diameter of the orifice and $\eta$ the viscosity of the fluid.

Since $R$ depends on $\rho$ as well as $\eta$, if its values are all so high that changing from one to another does not affect the shape of the jet, it follows that the shape is unaffected, not only by a change of viscosity caused by substituting one fluid for another, but also by a simultaneous change from one constant density to another. Hence at high values of $R$ the contraction coefficient will be the same in the supposed case of discharge of a gas at the constant density $\rho_{1}$ as it would be in the discharge of a liquid of that or of any other constant density; and the value of $\mu$ may therefore be determined by experiments on water at high values of $R$.

\section{BASIS FOR A NEW TREATMENT OF THE PROBLEM}

We assume that with given initial conditions $\left(p_{1}, \rho_{1}\right)$, and with a given mass flow $M_{1}$-which implies a particular linear speed $S_{1}$ along the pipe-the force $F_{0}$ exerted by the gas on the upstream face of the orifice plate is the same when the subsequent discharge through the orifice takes place isentropically, as if it went on with artificial cooling at constant density. This enables us to dispense with the assumption of uniform expansion beyond the plane of the orifice, and with several auxiliary approximations of which the importance is difficult to estimate.

The approximation to reality will be closer for small than for large values of $\left(p_{1}-p_{2}\right) / p_{1}$ and, probably, for small than for large values of $m$; and it is hardly to be expected that the results obtained from the 
assumption will agree well with experiment for large values of $m$ or for pressure ratios near the critical.

In so far as the assumption is valid, it permits of applying the momentum principle, and we proceed as follows:

\section{ISENTROPIC FLOW FROM $\left(p_{1}, \rho_{1}\right)$ TO $p_{2}$}

The equations of energy and continuity are

$$
S_{2}^{2}-S_{1}^{2}=\frac{2 \gamma}{\gamma-1} \cdot \frac{p_{1}}{\rho_{1}}\left(1-y^{\frac{\gamma-1}{\gamma}}\right)=\frac{2 p_{1} \phi}{\rho_{1} y^{\frac{1}{\gamma}}}
$$

and

whence

$$
M=A \rho_{1} S_{1}=\mu_{a} m A \rho_{2} S_{2}=\mu_{a} m A \rho_{1} y^{\frac{1}{\gamma}} S_{2}
$$

$$
S_{2}=\sqrt{\frac{2 p_{1} \phi}{\rho_{1} y^{\frac{1}{\gamma}}\left(1-\mu_{a}^{2} m^{2} y^{\frac{2}{\gamma}}\right)}}
$$

and

$$
M=\mu_{a} m A \rho_{1} y^{\frac{1}{\gamma}} \sqrt{\frac{2 p_{1} \phi}{\rho_{1} y^{\frac{1}{\gamma}}\left(1-\mu_{a}^{2} m^{2} y^{\frac{2}{\gamma}}\right)}}
$$

The time rate of increase of momentum is

and the force acting is

$$
M\left(S_{2}-S_{1}\right)=\frac{2 \mu_{a} m A p_{1} \phi}{1+\mu_{a} m y^{\frac{1}{\gamma}}}
$$

$$
F=A\left(p_{1}-m p_{2}\right)-F_{0}
$$

whence, equating (8) with (9) and dividing by $A p_{1}$, we have

$$
1-m y-\frac{F_{0}}{A p_{1}}=\frac{2 \mu_{a} m \phi}{1+\mu_{a} m y^{\frac{1}{\gamma}}}
$$

6. FLOW AT CONSTANT DENSITY FROM $\left(p_{1}, \rho_{1}\right)$ TO $p_{3}$ The equations of energy and continuity are

and

$$
S_{3}^{2}-S_{1}^{2}=2 \frac{p_{1}}{\rho_{1}}(1-w)
$$

whence

$$
M=A \rho_{1} S_{1}=\mu m A \rho_{1} S_{3}
$$

and

$$
S_{3}=\sqrt{\frac{2 p_{1}(1-w)}{\rho_{1}\left(1-\mu^{2} m^{2}\right)}}
$$

$$
M=\mu m A \rho_{1} \sqrt{\frac{2 p_{1}(1-w)}{\rho_{1}\left(1-\mu^{2} m^{2}\right)}}
$$


The rate of increase of momentum is

and the force acting is

$$
M\left(S_{3}-S_{1}\right)=\frac{2 \mu m A p_{1}(1-w)}{1+\mu m}
$$

whence by (15)

$$
F=A\left(p_{1}-m p_{3}\right)-F_{0}
$$

$$
1-m w-\frac{F_{0}}{A p_{1}}=\frac{2 \mu m(1-w)}{1+\mu m}
$$

\section{APPLICATION OF THE FUNDAMENTAL ASSUMPTION}

The assumption is that if equations (7) and (14) give the same value of $M$, the value of $F_{0}$ will be the same in (10) as in (17). Accordingly, we may eliminate the unknown force $F_{0}$ by subtracting (17) from (10) and after dividing by $m$ we have

$$
w-y=\frac{2 \mu_{a} \phi}{1+\mu_{a} m y^{\frac{1}{\gamma}}}-\frac{2 \mu(1-w)}{1+\mu m}
$$

Upon equating (7) and (14), removing the common factors, and squaring, we have

$$
\frac{\mu^{2}(1-w)}{1-\mu^{2} m^{2}}=\frac{\mu_{a}^{2} y^{\frac{1}{\gamma}} \phi}{1-\mu_{a}^{2} m^{2} y^{\frac{2}{\gamma}}}
$$

and after eliminating $w$ between (18) and (19) we have a quadratic equation of which the solution may be expressed in the form

$$
\frac{\mu_{a}}{\mu}=\frac{B}{A}\left(1-\sqrt{1-\frac{A Q}{B^{2}}}\right)
$$

where

$$
\left.\begin{array}{l}
A=y^{\frac{1}{\gamma}}\left[\left(2 \mu+\mu^{2} m^{2}-1\right) \phi-\mu^{2} m^{2} y^{\frac{1}{\gamma}}(1-y)\right] \\
B=\mu \phi \\
Q=1-y \\
\phi=\frac{\gamma}{\gamma-1} y^{\frac{1}{\gamma}}\left(1-y^{\left.\frac{\gamma-1}{\gamma}\right)}\right. \\
y=\frac{p_{2}}{p_{1}}
\end{array}\right\}
$$

The result embodied in equations (20) and (21) is to be compared with experiment and we have next to examine the available data.

\section{EXAMINATION OF THE EXPERIMENTAL DATA}

In the absence of accurate measurements of the diameter of the vena contracta, we have recourse to the values of discharge coefficients obtained from measurements of mass flow.

The "adiabatic" discharge coefficient $C_{a}$ for a gas is defined by

$$
M=C_{q} M_{i \cdot a}
$$


where $M$ is the observed mass flow and $M_{t \cdot a}$ is the "theoretical" mass flow found from equation (7) by ignoring the phenomena of contraction and setting $\mu_{a}=1$. Since $C_{a}$ is not constant, we may write

$$
C_{a}=C Y_{a}
$$

where $C$ is the limiting value which $C_{a}$ approaches as $p_{2} \doteq p_{1}$, and the "expansion factor" $Y_{a}$ is a function of $p_{2} / p_{1}$ which reduces to unity when $p_{2}=p_{1}$.

The discharge coefficient $C_{l}$ for a liquid is defined by

$$
M=C_{l} M_{t \cdot l}
$$

in which $M_{t . l}$ is the "theoretical" mass flow found by setting $\mu=1$ in equation (14). But if we set $\mu_{a}=\mu=1$ and $p_{3}=p_{2}$, equation (7) approaches (14) as a limiting form when $p_{2}=p_{1}$. Hence $C_{l}$ and $C$ are identical, and instead of attempting to find the value of $C$ by extrapolating to very small pressure differences from measurements of $C_{a}$, we may equally well find it from experiments on a liquid.

The stipulation that the Reynolds number shall be so high that its precise value is no longer important, implies that the dissipative resistances due to viscosity have become insignificant. The contraction coefficients $\mu_{a}$ and $\mu$ may therefore be regarded as sensibly identical with the discharge coefficients $C_{a}$ and $C$, and for the purpose of comparison with equation (20) we may set

$$
\frac{\mu_{a}}{\mu}=\frac{C_{a}}{C}=Y_{a}
$$

The experimental data suitable for a direct test of the formula are very few; for it was postulated that the discharge should take place into a space that was large enough for the static pressure in it to be uniform, and the only satisfactory experiments of this sort that have come to my attention are those of Bachmann ${ }^{4}$ on the discharge of air into the atmosphere through an orifice on the end of a pipe.

The internal diameter of the pipe was $8.25 \mathrm{~cm}$ and the diameter of the orifice was $2.003 \mathrm{~cm}$, so that the diameter ratio was $\beta=0.2428$ and the area ratio was $\beta^{2}=m=0.0590$. Of the 18 values of $C_{a}$ obtained at speeds below the critical, the one that was measured at the lowest rate of discharge and is therefore the least reliable is far out of line with the others. 'The remaining 17 are represented without systematic departures by the equation

$$
C_{a}=0.5989\left(1+0.2431 \frac{p_{1}-p_{2}}{p_{2}}\right)
$$

the greatest departure being less than 0.5 per cent and the mean departure less than 0.2 per cent.

The important thing for our present purposes is the expansion fuctor

$$
Y_{a}=1+0.2431 \frac{p_{1}-p_{2}}{p_{2}}
$$

\footnotetext{
'H. Bachwilann, "Beitrag zur Messung von Luftmengen," Dissertation, Darmstadt; 1911.
} 
but it is interesting to compare the ralue of $C$ with values obtained from orifices installed in pipes, with $p_{2}$ taken as the minimum pressure observed on the downstream side of the orifice.

Witte' $\mathrm{S}^{5}$ experiments on water flowing through perfectly squareedged orifices installed in smooth pipes, with the pressures taken at the face of the plate, gave values of $C$ which, for diameter ratios between $\beta=0.2$ and $\beta=0.75$, or $0.04<m<0.56$, are represented without systematic error by the equation

$$
\frac{C}{\sqrt{1-m^{2}}}=K=0.5983+0.395 m^{2}
$$

The maximum departure is about 0.25 per cent and the mean departure for the 17 orifices tested is less than 0.1 per cent, so that the representation by equation (28) is very good.

For Bachmann's area ratio, (28) gives $C=0.5986$, but this must be corrected to obtain the corresponding value which would have been found if the pressure difference had been measured between the upstream and downstream minima instead of between the two faces of the plate. Experiments on air which will be published in the near future show that this correction factor is about 0.9983 , so that we have from Witte's experiments on water $C=0.5976$ as compared with the value 0.5989 from Bachmann's work-an excellent agreement.

The equation adopted for representing the results of the experiments on compressed air, described in Bureau of Standards Research Paper No, $49,{ }^{6}$ namely

$$
C=0.5970+0.09 \mathrm{~m}^{2}
$$

gives $C=0.5973$ for Bachmann's value of $m$.

Next to Bachmann's data, the best at my disposal were obtained from experiments on natural gas, performed at Los Angeles in 1929 under the direction of H. S. Bean of the Bureau of Standards, as part of an investigation being carried on by the Natural Gas Division of the American Gas Association, in cooperation with the Bureau of Standards. The circumstances were more favorable as regards steadiness of flow than in the experiments on compressed air just referred to, so that the precision was higher and the results are preferable.

The orifices were installed in straight pipes, as in Witte's experiments on water, so that the discharge space was restricted and the static pressure in it not entirely uniform. It is to be presumed, however, that if the area ratio $m$ is small, and if the minimum pressure, observed at the wall of the pipe on the discharge side is taken as the value of $p_{2}$, the arrangement will correspond fairly closely with that presupposed in developing equation (20); and the agreement noted above between Witte's and Bachmann's results lends support to this presumption.

New commercial steel pipes of 4,8 , and 16 inches nominal diameter were employed, and a honeycomb of smaller pipes was placed in each, from 10 to 15 pipe diameters ahead of the orifice. The orifices (23 in number) had diameter ratios ranging from 0.124 to 0.869 , but the 
data to be considered here are only those obtained with the 14 orifices for which the diameter ratios were between 0.3 and 0.75 , or 0.09 $<m<0.56$. For, as is the case with Witte's values of $C$ for water, the variations of $C$ can be more accurately represented by simple empirical equations within these limits than for $\beta<0.2$ or $>0.75$.

The results are expressed in the form

$$
C_{1}=f(x)
$$

where $x=\left(\mathrm{p}_{1}-p_{2}\right) / p_{1}$ and $C_{1}$ is defined by the equation

$$
M=C_{1} m A \sqrt{\frac{2 \rho_{1}\left(p_{1}-p_{2}\right)}{1-m^{2}}}
$$

$M$ being the actual mass flow. The coefficient $C_{1}$ is connected with $C_{a}$ by the relation

where

$$
C_{1}=C_{a} Z_{1} Z_{2}
$$

$$
z_{1}=\sqrt{\frac{\gamma y^{\frac{2}{\gamma}\left(1-y^{\frac{\gamma-1}{\gamma}}\right)}}{(\gamma-1)(1-y)}}
$$

and

$$
z_{2}=\sqrt{\frac{1-m^{2}}{1-m^{2} y^{\frac{2}{\gamma}}}}
$$

Since $z_{1} \doteq z_{2} \doteq 1$ when $p_{2} \doteq p_{1}$ or $y \doteq 1, C_{1}$ and $C_{a}$ have the same limiting value $C$ and we may write

$$
C_{1}=C Y_{1}
$$

in which $Y_{1}$, like $Y_{a}$, is a function of $x$ which reduces to unity when $x=0$. By equations (32) and (34) we have

$$
C_{a}=C Y_{a}=\frac{C_{1}}{z_{1} z_{2}}=C \frac{Y_{1}}{z_{1} z_{2}}
$$

whence

$$
Y_{a}=\frac{Y_{1}}{z_{1} z_{2}}
$$

and if $Y_{1}$ is known, the value of $Y_{a}$ for use in equation (25) may be found from it.

When the values of $C_{1}$ for each of the 14 orifices are plotted against the values of $x$ at which they were measured, the points lie along a straight line, within the experimental errors. The slope of the line varies with $m$, and the average result for all the orifices is represented by the equation

$$
Y_{1}=1-\left(0.32+0.3 m^{2}\right) x
$$

In order to use this result in connection with Bachmann's values of $Y_{a}$ for air, an allowance must be made for the difference between 
$\gamma=1.40$ for air and $\gamma=1.284$, computed from the composition of the Los Angeles gas. For this purpose I have assumed the relation

$$
C_{1}=f\left(\frac{x}{\gamma}\right)
$$

proposed by E. S. Smith, ${ }^{7}$ which appears to be accurately true for low values of $x$, and to be a good approximation even as far as the critical pressure ratio.

On the basis of Smith's relation, equation (37) is to be regarded as a special form of the more general equation

$$
Y_{1}=1-\left(0.41+0.39 m^{2}\right) \frac{x}{\gamma}
$$

and for the case of air, with $\gamma=1.40$, this reduces to

$$
Y_{1}=1-\left(0.293+0.28 m^{2}\right) x
$$

For the purpose of comparison with Bachmann's data on air and with equation (20), equation (39) will be assumed to represent the results that would have been obtained at Los Angeles if the experiments had been made with air instead of natural gas.

\section{COMPARISON WITH THE NEW FORMULA}

Since the computations required are tedious, they have been carried out only for a few simple values of $m^{2}$ and of $p_{2} / p_{1}$, and the experimental values of $Y_{a}$ have not been treated individually but taken from the empirical equations which represent them.

Bachmann's results have been taken from equation (27) or

$$
Y_{a}=1+0.2431 \frac{p_{1}-p_{2}}{p_{2}}
$$

which represents them within the experimental errors. In accordance with (36) and (39), the equation

$$
Y_{a}=\frac{1-\left(0.293+0.28 m^{2}\right) x}{z_{1} z_{2}}
$$

has been taken to represent the results of the experiments at Los Angeles.

The values of $\mu=C$ required for substitution in equations $(20,21)$ were computed from equation (28), which represents Witte's experimental results, and then corrected to what they would have been if the pressures had been observed at the upstream and downstream minima, by means of factors determined from the experiments on air already referred to.

7 The proposal was made in 8 paper presented to the World Engineering Congress, Tokio, 1929, and subsequently to the Annual Meeting of the American Society of Mechanical Engineers, New York, December, 1929. The introduction of the "expansion factor" $Y$ is also due to Mr. Smitb. 
In addition to computing values of $\mu_{a} / \mu$ from (20), I have also made corresponding computations by means of the equation

$$
\frac{\mu_{a}}{\mu}=\left[\frac{\left(1-\mu^{2}\right) \frac{p_{1}}{p_{2}}-\mu^{2}\left(1-m^{2}\right)}{1-m^{2} \mu^{2}}\right]^{\frac{2}{3 \gamma}}
$$

deduced by Witte ${ }^{8}$ from the assumption of uniform expansion.

The results are summarized in the following table.

\begin{tabular}{|c|c|c|c|c|c|c|c|}
\hline$m^{2}$ & $\sqrt{m}=\beta$ & $\mu$ & $\frac{p_{2}}{p_{1}}$ & $\begin{array}{c}\mathrm{Y}_{a} \\
\text { Bachmann } \\
\text { equation } \\
(40)\end{array}$ & $\begin{array}{c}Y_{a} \\
\text { Los Angeles } \\
\text { equation } \\
(41)\end{array}$ & $\begin{array}{c}\frac{\mu_{a}}{\mu} \\
\text { equation } \\
(20)\end{array}$ & $\underset{\substack{\text { equation } \\
(42)}}{ }$ \\
\hline 1 & 2 & 3 & 4 & 5 & 6 & $\gamma$ & 8 \\
\hline $\begin{array}{l}0.005 \\
(.00348)\end{array}$ & $\begin{array}{l}0.266 \\
(.243)\end{array}$ & 0.5970 & $\begin{array}{l}0.9 \\
.8 \\
.7 \\
.6 \\
.53\end{array}$ & $\begin{array}{l}1.027 \\
1.061 \\
1.104 \\
1.162 \\
1.216\end{array}$ & $\begin{array}{l}\text { 1. } 028 \\
\text { 1. } 062 \\
\text { 1. } 107 \\
\text { 1. } 167 \\
\text { 1. } 222\end{array}$ & $\begin{array}{l}\text { 1. } 030 \\
\text { 1. } 066 \\
\text { 1. } 112 \\
\text { 1. } 170 \\
1.221\end{array}$ & $\begin{array}{l}1.032 \\
1.068 \\
1.108 \\
1.155 \\
1.190\end{array}$ \\
\hline .02 & .376 & .5975 & $\begin{array}{l}.9 \\
.8 \\
.7 \\
.6 \\
.53\end{array}$ & & $\begin{array}{l}1.028 \\
1.064 \\
1.109 \\
1.170 \\
1.225\end{array}$ & $\begin{array}{l}\text { 1. } 030 \\
1.067 \\
1.112 \\
1.170 \\
1.222\end{array}$ & $\begin{array}{l}1.032 \\
1.068 \\
1.109 \\
1.155 \\
1.191\end{array}$ \\
\hline $1+1$ & 1 & & .9 & & 1. 030 & 1. 030 & 1. 033 \\
\hline .06 & .495 & .6003 & $\begin{array}{l}.8 \\
.7 \\
.6 \\
.53\end{array}$ & & $\begin{array}{l}1.067 \\
1.115 \\
1.177 \\
1.233\end{array}$ & $\begin{array}{l}1.067 \\
1.113 \\
1.172 \\
1.223\end{array}$ & $\begin{array}{l}1.069 \\
1.110 \\
1.155 \\
1.192\end{array}$ \\
\hline .24 & .700 & .6038 & $\begin{array}{l}.9 \\
.8 \\
.7 \\
.6 \\
.53\end{array}$ & & $\begin{array}{l}\text { 1. } 042 \\
\text { 1. } 091 \\
\text { 1. } 149 \\
\text { 1. } 220 \\
\text { 1. } 282\end{array}$ & $\begin{array}{l}\text { 1. } 033 \\
\text { 1. } 072 \\
\text { 1. } 121 \\
\text { 1. } 182 \\
1.235\end{array}$ & - \\
\hline
\end{tabular}

Columns 1 and 2 give the values of $m^{2}$ for which the computations were carried out, and the corresponding values of the diameter ratio $\beta$. The figures in parentheses refer to Bachmann's orifice; they do not differ enough from the figures above them to affect the values in columns 6 to 8 .

Column 3 gives the values assumed for $\mu$ in equation (20) and column 4 shows the values used for the pressure ratio $p_{2} / p_{1}$. The remaining columns of the table are sufficiently explained by their headings.

\section{CONCLUSIONS}

1. Comparison of columns 5 and 6 of the table shows that the empirical equation adopted for representing the average results of the Los Angeles experiments on natural gas, when reduced by means of Smith's assumption that $C_{1}=f(x / \gamma)$, agrees with the results of Bachmann's measurements on air within 0.5 per cent, the systematic difference being within the precision of the Los Angeles experiments.

See fontnoto 1, p. 766. 
The assumption proposed by Hodgson, ${ }^{9}$ namely

$$
C_{1}=f\left[\left(\frac{p_{2}}{p_{1}}\right)^{\frac{1}{\gamma}}\right]
$$

while not so simple as Smith's relation seems better justified. If the reduction of the Los Angeles results from $\gamma=1.284$ to $\gamma=1.400$ is made in accordance with this equation, the greatest difference between columns 5 and 6 is decreased from 0.006 to 0.002 .

2. Comparison of columns 6 and 7 shows that the new formula agrees with the Los Angeles results to within 0.5 per cent up to $\beta=0.376$, and to within 0.8 per cent at $\beta=0.495$. For $\beta=0.7$ the agreement is poor throughout, as might be expected; for with so large a diameter ratio the condition of discharge in to a pipe is extremely different from the free discharge postulated in deducing the formula.

3. Comparison of columns 6 and 8 shows that Witte's formula (42) represents the facts, as far as they are known, nearly as well as the new formula, for pressure ratios down to about 0.7 , but that it is inferior for still lower values of $p_{2} / p_{1}$.

Washington, February 16, 1931.

Discussion of papers on Fluid Flow presented at the annual meeting of the A. S. M. E., New York, December, 1929. 